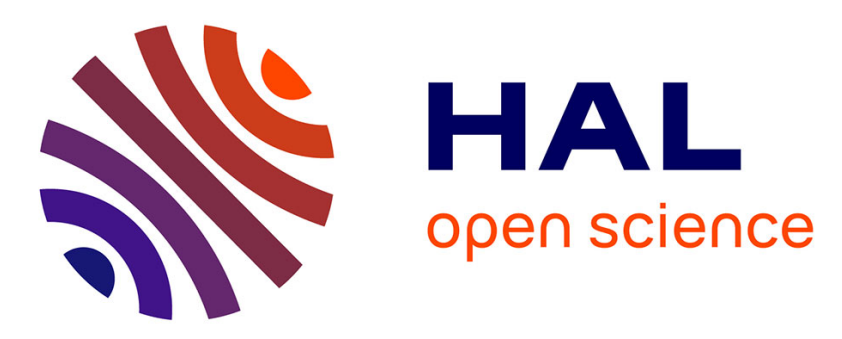

\title{
On the influence of sample length and measurement noise on the stochastic subspace damage detection technique
}

Saeid Allahdadian, Michael Döhler, Carlos Ventura, Laurent Mevel

\section{- To cite this version:}

Saeid Allahdadian, Michael Döhler, Carlos Ventura, Laurent Mevel. On the influence of sample length and measurement noise on the stochastic subspace damage detection technique. IMAC - 34th International Modal Analysis Conference, Jan 2016, Orlando, FL, United States. 10.1007/978-3-31929956-3_4. hal-01262256

\section{HAL Id: hal-01262256 \\ https://hal.inria.fr/hal-01262256}

Submitted on 26 Jan 2016

HAL is a multi-disciplinary open access archive for the deposit and dissemination of scientific research documents, whether they are published or not. The documents may come from teaching and research institutions in France or abroad, or from public or private research centers.
L'archive ouverte pluridisciplinaire HAL, est destinée au dépôt et à la diffusion de documents scientifiques de niveau recherche, publiés ou non, émanant des établissements d'enseignement et de recherche français ou étrangers, des laboratoires publics ou privés. 


\title{
On the influence of sample length and measurement noise on the stochastic subspace damage detection technique
}

\author{
Saeid Allahdadian ${ }^{\dagger, a}$, Michael Döhler ${ }^{\psi, b}$, Carlos E. Ventura ${ }^{\dagger, c}$ and Laurent Mevel ${ }^{\psi, d}$ \\ ${ }^{\dagger}$ University of British Columbia, Vancouver, Canada \\ ${ }^{\psi}$ Inria/IFSTTAR, I4S, Campus de Beaulieu, 35042 Rennes, France \\ ${ }^{a}$ saeid@civil.ubc.ca, ${ }^{b}$ michael.doehler@inria.fr, ${ }^{c}$ ventura@civil.ubc.ca, ${ }^{d}$ laurent.mevel@inria.fr
}

\begin{abstract}
In this paper the effects of measuring noise and number of samples is studied on the stochastic subspace damage detection (SSDD) technique. In this technique, i.e., SSDD, the need of evaluating the eigenstructure of the system is circumvented, making this approach capable of dealing with real-time measurements of structures. In previous studies, the effect of these practical parameters was examined on simulated measurements from a model of a real structure. In this study, these effects are formulated for the expected damage index evaluated from a Chi-square distributed value. Several theorems are proposed and proved. These theorems are used to develop a guideline to serve the user of the SSDD method to face these effects.
\end{abstract}

Keywords: damage detection, subspace method, health monitoring, signal noise, sampling, stochastic subspace method

\section{Introduction}

Structural health monitoring is regarded as the main tool in assessing the functionality of existing structures. The importance of these techniques and researches becomes obvious by considering that failure of a structure can result in catastrophic loss. Existing civil structures deteriorate by aging and under different loading conditions imposed from natural phenomena such as earthquakes, typhoons, flood and etc. Therefore, it is imperative to investigate the safety of continuing using these structures, especially after occurring major demands on the structure from these phenomena.

Numerous researches can be found in the literature and different approaches are proposed to deal with this problem by detecting possible damages in a structure. Some of these tests include sampling of the structure, which may affect the functionality of structure. These tests are named destructive tests. However the other type of the tests, namely non-destructive tests, do not involve with any action that can damage the structure or affect its functionality. Due to the need of continuation of the serviceability of the structure, more researchers have been focusing on the latter approach.

Nondestructive damage detection techniques can be categorized into two groups based on their requirements [1-2]: (I) local techniques, which need access to all parts of the structure or the location of damage if known, and (II) global damage techniques which use vibration data to evaluate global dynamic characteristics of the structure. Employing the local techniques may lead to interference in the operation of the structure and is not suitable for major structures. However, in the latter method there is no need to know or have access to the location of damage in priori.

The global techniques can be also categorized into two groups based on their approach to the problem. In the first category, the structural properties are identified and employed to assess the condition of the structure. The structural properties identified from these approaches include stiffness, damping, mass, load paths and boundary conditions (supports, connections, etc.). In the second category, the eigen-structure of the problem is employed to evaluate the safety condition of the structure. In these methods, modal properties such as natural frequencies, modal damping values and mode shapes are used to identify any changes in the structure. Any change in the structural properties leads to a change in the modal parameters of the structure. However generally, identifying the modal parameters in a structure is more practical and accurate than the structural properties.

In order to keep the structure in operation, shaking the structure artificially or using impact loads are not promising. Therefore by employing ambient vibration testing, the operation of the structure will not be interfered. In this case due to the fact that the input excitation to the structure, e.g. wind, traffic, earth vibration, cannot be measured practically, output-only damage detection 
techniques are of interest. Moreover, the process of evaluating and matching the modal parameters of a structure is also time consuming [3] and it usually cannot be employed in real-time monitoring of structures which are not well instrumented. In addition, local damage in a structure affects typically on higher frequency modes $([2,4])$ which are not usually identifiable to be used in damage detection due to their high modal density and low participation factors [5]. Evaluation of these modal characteristics can be avoided by using output-only statistical approaches e.g. Kalman filter technique [6], outlier analysis method [7] or the stochastic subspace damage detection technique (SSDD) ([8-9]).

The SSDD technique evaluates the global condition of a structure by identifying changes in the eigen-structure of the system. The damage can be detected by comparing a statistical model from the possibly damaged structure to thresholds obtained from a reference state. A subspace based residual function between these states is defined and compared using a $\chi^{2}$ test. The results from $\chi^{2}$ test can be displayed and monitored in a chart, namely control chart [10]. Therefore, there is no need to estimate the natural frequencies and mode shapes, making this approach capable of being used in real-time monitoring of structures. In this way, the whole eigen-system of the measurements are included in the damage detection and the focus is not only on dominant frequencies. Including higher modes in this evaluation makes the damage detection approach more robust, considering that the main effect of local damages is on higher mode shapes.

Two main challenges in health monitoring of real structures are low number of sensors and existence of noise in the measurements. Statistical damage detection methods including SSDD have a robust architecture that can deal with sparsely instrumented structures, at least for the level one of damage detection, namely investigating the existence of damage. Moreover, these methods can also deal with noisy data due to their statistical approach to the problem and that the mean of the noise is usually zero in the time domain. However, this effect needs to be studied in detail for these damage detection techniques.

In this study we focus on the effect of measurement noise and number of samples, i.e. measurement length, on the SSDD technique. Existence of noise in experimental data is inevitable. There are different sources of the noise in measuring a structure [11] such as the change in excitation sources [12], noise of measuring instruments and human error. Moreover, the data quality (noise ratio) can affect significantly on the damage detection output (e.g. [13]). Therefore, investigating the effect of this inherent characteristic of the measurements on the SSDD technique is an important factor in assessing its functionality. It was demonstrated that SSDD technique can perform robustly under ambient excitations with changing statistics [11,14]. In our previous studies, the effect of measurement noise ratio [15-16], type of element damaged [17] and number of samples [16] were studied briefly by examining it for a model of real bridge, the S101 Bridge. It was shown that this technique can deal with even very high ratios of noise in the data.

In this paper the objective is to analyze the theory associated to the effects of the measurement noise and number of samples on this technique. This study helps in having a better understanding of the results from the SSDD approach and in creating some guidelines for using it.

This paper is organized as follows. In Section 2, the SSDD approach is recalled. In section 3, a theoretical analysis of its properties regarding measurement noise and sample length is carried out. Section 4 contains a numerical validation of the theoretical results and concluding remarks are presented in Section 5.

\section{Stochastic subspace damage detection technique}

The theories and formulations of stochastic subspace damage detection (SSDD) stem from the subspace based system identification. In this section, models, parameters and formulations needed to derive the final residual used in assessing the condition of the system is presented based on studies in [8-9].

\subsection{Dynamic equilibrium equation in discrete time domain}

The state-space representation of a dynamic system is well known. Herein, the governing equation for the dynamic behaviour of a structural system is presented and then it is reformed to the state-space representation. The dynamic behaviour of a structure can be modeled with the following formulation

$$
\left\{\begin{array}{l}
M \ddot{u}(t)+C \dot{u}(t)+K u(t)=p(t) \\
y(t)=L \ddot{u}(t)+e(t)
\end{array}\right.
$$

where $M, C$ and $K$ are mass, damping and stiffness matrices, respectively, and $u$ represents the displacement vector in all degrees of freedom of the system. Vector $p$ also shows the vector of forces and $t$ denote continuous time. It should be noted that the external force $p$ is unknown while it is assumed to be a non-stationary white noise. Vector $y$ also contains the output responses measured from the structure. Based on the type of the sensor recording acceleration, velocity or displacement the 
second part of the equation changes; herein the type of the sensor is assumed as accelerometers. Matrix $L$ states the location of the sensors in relation to the geometry of the degrees of freedom, and $e$ represents the measurement noise.

The discrete time state-space form of model (1) can be written by performing sampling with time step $\tau$ as

$$
\left\{\begin{array}{c}
x_{k+1}=F x_{k}+w_{k} \\
y_{k}=H x_{k}+v_{k}
\end{array}\right.
$$

where, the state is represented by $x \in \mathbb{R}^{n}$ and the measured output is $y \in \mathbb{R}^{r} . r$ is the number of sensors and $n$ is the system order. The state transition matrix is represented by $F$, and $H$ shows the observation matrix with dimensions $n \times n$ and $r \times n$, respectively. The state noise $w_{k}$ and output noise $v_{k}$ are assumed to be white noise. The state $x$ and the measured output, $y$, are related to the displacement vector with equation (3).

$$
x_{k}=\left[\begin{array}{c}
u(k \tau) \\
\dot{u}(k \tau)
\end{array}\right], \quad y_{k}=y(k \tau)
$$

The modal parameters of the dynamic model (1), which are present in its eigenvalues $\mu$, and mode shapes, $\Psi$, can be related to the eigenvalues $\lambda$ and eigenvectors $\phi$ of the state transition matrix, $F$ :

$$
\left\{\begin{array}{l}
\lambda=e^{\mu \tau} \\
\varphi=\psi
\end{array} \quad \text { where } \varphi=H \phi \text { and } \psi=L \Psi\right.
$$

The canonical parameterization of system (2) is formed by pairs $(\lambda, \varphi)$ which is referred as the system eigenstructure and is employed as the system parameter $\theta \in \mathbb{C}^{n(r+1)}$ defined as

$$
\theta=\left[\begin{array}{c}
\Lambda \\
\operatorname{vec}(\Phi)
\end{array}\right]
$$

in which $\Lambda$ is the vector containing all the eigenvalues $\lambda$ and $\Phi$ is the matrix composed of all eigenvectors $\phi$.

\subsection{Output-only covariance based subspace system identification}

In order to compute a residual vector between the reference and the current states of the system, the output-only covariance based subspace system identification method [18] is employed. By defining the output covariance as $R_{i}=\mathbf{E}\left(y_{k} y_{k-i}^{T}\right)$, the block Hankel matrix $\mathbf{H}_{p}$ can be composed as

$$
\mathbf{H}_{p}=\left[\begin{array}{llll}
R_{1} & R_{2} & \cdots & R_{p} \\
R_{2} & R_{3} & \cdots & R_{p+1} \\
\vdots & \vdots & \ddots & \vdots \\
R_{p+1} & R_{p+2} & \cdots & R_{2 p}
\end{array}\right]=\operatorname{Hank}\left(R_{i}\right)
$$

The output covariances satisfy $R_{i}=H F^{i-1} G$ [19], where $G=\mathbf{E}\left(x_{k+1} y_{k}^{T}\right)$ is the cross covariance between the states and the outputs, which leads to the well-known factorization property of

$$
\mathbf{H}_{p}=\mathrm{O}_{p} \mathrm{C}_{p}
$$

where

$$
\mathrm{O}_{p}=\left[\begin{array}{c}
H \\
H F \\
\vdots \\
H F^{p}
\end{array}\right], \quad \mathrm{C}_{p}=\left[\begin{array}{llll}
G & F G \cdots F^{p-1} G
\end{array}\right] .
$$


The observation matrix, $H$, state transition matrix, $F$, and subsequently the system parameters, $\theta$, can be computed from the defined observability matrix, $\mathrm{O}_{p}$. The residual employed in damage detection is directly linked to $\mathrm{O}_{p}$ and thus there is no need to identify the system matrices and parameters.

\subsection{Residual vector formation}

By assuming that the system parameter in reference state of the structure is $\theta_{0}$ and in current state is $\theta$, a residual function is defined between these states which reacts to the changes in the system due to, for instance, damage. In order to create such a residual, the left null-space of the observability matrix $\mathrm{O}_{p}$, namely orthonormal matrix $S$, is computed from performing e.g. a singular value decomposition. The reference state $\theta=\theta_{0}$ is then characterized by

$S^{T}\left(\theta_{0}\right) \mathrm{O}_{p}\left(\theta_{0}\right)=0$.

Due to the factorization property (6), the left null-space of $\mathbf{H}_{p}$ is equal to $S\left(\theta_{0}\right)$ and hence (8) can be rewritten as

$S^{T}\left(\theta_{0}\right) \mathbf{H}_{p}=0$.

With the interpretation that if the system is damaged the system parameter $\theta$ becomes changed, i.e. $\theta \neq \theta_{0}$, two hypotheses are defined as following.

$\begin{cases}H_{0}: & \theta=\theta_{0} \therefore \text { unchanged system } \\ H_{1}: & \theta \neq \theta_{0} \therefore \text { changed system (damaged) }\end{cases}$

To test these hypothesis, a residual function needs to be defined based on property (9) that holds if and only if $\mathbf{H}_{p}$ corresponds to the reference state. Since matrix $S\left(\theta_{0}\right)$ depends implicitly on parameter $\theta_{0}$ (we are treating it as a function of $\theta_{0}$ [9]), a representation of the current state parameter of the structure, i.e. $\theta$ is needed. Therefore, by measuring data from the current state of the structure, an estimation of the block Hankel matrix, i.e. $\hat{\mathbf{H}}_{p}$, is computed from their covariances as

$\hat{R}_{i}=\frac{1}{N} \sum_{k=1}^{N} y_{k} y_{k-i}^{T}, \quad \hat{\mathbf{H}}_{p}=\operatorname{Hank}\left(\hat{R}_{i}\right)$

In view of (9), this empirical block Hankel matrix is used to create residual function (12) which corresponds to the difference between $\theta$ and $\theta_{0}[8-9]$.

$\zeta_{N}^{e}=\sqrt{N} \operatorname{vec}\left(S\left(\theta_{0}\right)^{T} \hat{\mathbf{H}}_{p}\right)$

The indexes $N$ and $e$ represent the number of samples and measurement noise level in the measured data, respectively. A change in the system parameter can be formulated based on the asymptotic local approach for change detection [20] as $\theta=\theta_{0}+\delta \theta / \sqrt{N}$ where $\delta \theta$ is defined as the (unknown) parameter change vector normalized by $\sqrt{N}$. Using this basis, the asymptotical distribution of the residual function fulfills the Central Limit Theorem (CLT), and is for $N \rightarrow \infty$

$$
\zeta_{N}^{e} \rightarrow \begin{cases}\mathcal{N}\left(0, \Sigma^{e}\right) & \text { under } H_{0} \\ \mathcal{N}\left(J^{e} \delta \theta, \Sigma^{e}\right) & \text { under } H_{1}\end{cases}
$$

in which $\Sigma^{e}$ is the asymptotic covariance and $J^{e}$ is the asymptotic sensitivity of the residual. In order to test these hypothesis, a generalized likelihood ratio (GLR) test is employed [8], which will be presented in next section.

\subsection{Hypothesis test}

\subsubsection{Parametric Chi-square test}

The GLR test for hypothesis (10) can be written as 


$$
\operatorname{GLR}\left(\zeta_{N}^{e}\right)=-2 \log \frac{L\left(\zeta_{N}^{e} \mid \theta_{0}\right)}{\sup _{\theta \in H_{1}} L\left(\zeta_{N}^{e} \mid \theta\right)}
$$

where $L(\bullet)$ represents the likelihood function. Plugging in the residual distributions from (13), it boils down to the test variable $[8,11]$

$$
\chi_{N}^{2}=\left(\zeta_{N}^{e}\right)^{T}\left(\Sigma^{e}\right)^{-1} J^{e}\left(\left(J^{e}\right)^{T}\left(\Sigma^{e}\right)^{-1} J^{e}\right)^{-1}\left(J^{e}\right)^{T}\left(\Sigma^{e}\right)^{-1} \zeta_{N}^{e} .
$$

which is asymptotically $\chi^{2}$-distributed with $d=\operatorname{rank}\left(J^{e}\right)=\operatorname{dim}(\theta)$ degrees of freedom. Its non-centrality parameter is 0 under $H_{0}$ and $\delta \theta^{T}\left(J^{e}\right)^{T}\left(\Sigma^{e}\right)^{-1} J^{e} \delta \theta$ under $H_{1}$.

The test variable in (15) is the parametric representation of a damage index and can be used to evaluate thresholds of safety, since its distribution shifts with the given non-centrality parameter under $H_{1}$. If the test value surpasses these thresholds, then it shows that the condition of the structure is being changed.

\subsubsection{Non-parametric Chi-square test}

By computing a null-space from a reference data set, a non-parametric residual is created for which there is no need to have a parametric model and to evaluate its parameters. Therefore, no system identification is needed. This null-space $S_{0}$ can be obtained by a singular value decomposition of the estimated Hankel matrix from the measurement data in reference state [21]. Similar to characterization in (8) and (9) it holds in the reference state:

$S_{0}^{T} \hat{\mathbf{H}}_{p}^{0}=0$.

$S_{0}$ and $\hat{\mathbf{H}}_{p}^{0}$ are, respectively, the estimated null-space and block Hankel matrix computed over a reference dataset. After measuring data from a possibly damaged structure, the block Hankel matrix is determined from the data and the residual is defined as

$$
\zeta_{N}^{e}=\sqrt{N} \operatorname{vec}\left(S_{0}^{T} \hat{\mathbf{H}}_{p}\right) \text {. }
$$

Since no explicit system parameterization is used, we have $J^{e}=\mathbf{I}$ in the residual distribution in (13), where $\mathbf{I}$ is the identity matrix, and the CLT (13) can be expressed as

$$
\zeta_{N}^{e} \rightarrow \begin{cases}\mathcal{N}\left(0, \Sigma^{e}\right) & \text { under } H_{0} \\ \mathcal{N}\left(\delta, \Sigma^{e}\right) & \text { under } H_{1}\end{cases}
$$

where $\delta$ is now directly linked to the change in the residual vector (when normalized by $\sqrt{N}$ ). Then, the test variable simplifies to

$\chi_{N}^{2}=\left(\zeta_{N}^{e}\right)^{T}\left(\Sigma^{e}\right)^{-1} \zeta_{N}^{e}$.

Analogously to the previous section, this variable is asymptotically $\chi^{2}$-distributed with $d=\operatorname{dim}\left(\zeta_{N}^{e}\right)$ degrees of freedom. Its non-centrality parameter is 0 under $H_{0}$ and $\delta^{T}\left(\Sigma^{e}\right)^{-1} \delta$ under $H_{1}$

For simplicity, this non-parametric test variable will be used in the following.

\section{Investigating the effect of noise and number of samples}

The residual $\zeta_{N}^{e}$ is a function of number of samples and the noise in the measured data. The dependence of this variable on the number of samples is explicit in equation (12). Moreover, analogous to the effect of change in the excitation properties [11], additional measurement noise superposed on the measured data will affect the cross covariance between the outputs, and therefore the estimated Hankel matrix. Thus, the evaluated residual (12) and its covariance $\Sigma^{e}$ are a function of the superposed noise. 
Hence, both the number of samples and measurement noise can change the residual and the final evaluated $\chi^{2}$ value. In this section their effect on the non-parametric $\chi^{2}$ test is studied for a constant damage.

It is always assumed that the residual covariance $\Sigma^{e}$ is estimated once on healthy data from the reference state of the structure, where usually lots of data is available allowing for a good covariance estimate [11]. The covariance is never recalculated when testing a residual $\zeta_{N}^{e}$ for damage that is computed on new test data.

Before starting the analysis, we recall a basic property of the $\chi^{2}$ distribution: let $\gamma$ be a $\chi^{2}$ distributed variable, $d$ its number of degrees of freedom and $n_{c}$ its non-centrality parameter. Then,

$\mathbf{E} \gamma=d+n_{c}$.

\subsection{Effect of number of samples}

The effect of number of samples can be seen in residual (17) both explicitly in terms of $\sqrt{N}$ and implicitly e.g. its variance and the change in the system parameter. The reason of pre-multiplying the square root of number of samples in the residual vector is that based on the Central Limit Theorem, the resultant product, i.e. (17), is distributed asymptotically normal as stated in (13) and (18), with its covariance being independent of the number of samples. Moreover, this framework allows for a tradeoff between number of samples and damage size: the $\chi^{2}$ test variable may have the same value either using a longer dataset with a smaller damage, or using a shorter dataset with a bigger damage. This also means that for constant (non-zero) damage the test variable grows with the number of samples. A detailed analysis is made in this section.

\subsubsection{Effect on the residual covariance}

Since the asymptotic residual covariance is the same in reference and damaged states (see Eq. (13),(18)), an estimate $\hat{\Sigma}^{e}$ of the covariance matrix $\Sigma^{e}$ is more conveniently obtained from data in the reference state of the structure under the assumption of no changes in the noise properties of the system [11]. The computation of the covariance estimate is described in detail in [11]. Note that the asymptotic covariance $\Sigma^{e}$ is independent of the number of samples $N$, which can also be seen in the CLT (13) and (18). Hence, the expected value of the covariance estimate $\hat{\Sigma}^{e}$ neither depends on the number of datasets nor their length used in the estimation. Of course, the quality of the estimate improves when using more data, and we assume that sufficient data has been used to achieve an accurate estimate.

\subsubsection{Effect on the $\chi^{2}$ test value}

Due to the CLT (18), the residual is approximately Gaussian for any fixed number of samples $N$, and it holds

$$
\zeta_{N}^{e} \approx\left\{\begin{array}{ll}
\mathcal{N}\left(0, \Sigma^{e}\right) & \text { under } H_{0} \\
\mathcal{N}\left(\delta, \Sigma^{e}\right) & \text { under } H_{1}
\end{array},\right.
$$

where $\delta=\sqrt{N} \Upsilon^{e}$ with $\Upsilon^{e}=\mathbf{E}\left(\operatorname{vec}\left(S_{0}^{T} \hat{\mathbf{H}}_{p}\right)\right)=\operatorname{vec}\left(S_{0}^{T} \mathbf{H}_{p}\right)$.

Note that $\Upsilon^{e}$ depends on the expected value $\mathbf{H}_{p}$ of the Hankel matrix of the current system (which is independent of the number of samples $N$ ), and $\hat{\mathbf{H}}_{p}$ is a consistent estimate of matrix $\mathbf{H}_{p}$. Note also that $\Upsilon^{e}=0$ if the system is in the reference state due to the definition of the null-space.

In the following, the influence of $N$ on the expected value of the test variable $\chi_{N}^{2}$ in (19) is investigated.

Theorem 1 Under the undamaged state of the structure, i.e. $H_{0}$ is true, increase or decrease of the number of samples does not change the mean of the $\chi^{2}$ value. 
Proof 1 Since $\zeta_{N}^{e} \approx \mathcal{N}\left(0, \Sigma^{e}\right)$ under $H_{0}$ (independently of the number of samples $N$ ), the non-centrality parameter of the resulting test variable $\chi_{N}^{2}$ in (19) is 0, as stated in Section 3. From the property (20) of the $\chi^{2}$ distribution it follows $\mathbf{E} \chi_{N}^{2}=d$ where $d=\operatorname{dim}\left(\zeta_{N}^{e}\right)$ is the number of degrees of freedom of $\chi_{N}^{2}$, independently of $N$.

Theorem 2 If the structure is damaged, i.e. $H_{1}$ is true, change of the number of samples will result in a change (in the same direction) in the mean of the evaluated $\chi^{2}$ test variable.

Proof 2 Under $H_{1}$ the non-centrality parameter of $\chi_{N}^{2}$ is $\delta^{T}\left(\Sigma^{e}\right)^{-1} \delta$. Since $\delta=\sqrt{N} \Upsilon^{e}$, the non-centrality parameter yields $N\left(\Upsilon^{e}\right)^{T}\left(\Sigma^{e}\right)^{-1} \Upsilon^{e}$, where both $\Upsilon^{e}$ and $\Sigma^{e}$ are independent of $N$. From the property (20) of the non-central $\chi^{2}$ distribution, it follows $\mathbf{E} \chi_{N}^{2}=d+N\left(\Upsilon^{e}\right)^{T}\left(\Sigma^{e}\right)^{-1} \Upsilon^{e}$. Thus the mean of the test variable grows (or decreases) when the number of samples of the same damaged system grows (or decreases).

\subsection{Effect of measurement noise}

Effect of the amount of measurement noise is investigated in two settings. In the first one, the properties of the measurement noise are the same in the reference state and possibly damaged state, while in the second setting they are different. Each of these settings are investigated in the following sections.

First, some properties regarding the noise properties of the state space system (2) are recalled [19]. They are given by

$$
\mathbf{E}\left[\left(\begin{array}{c}
w_{k} \\
v_{k}
\end{array}\right)\left(\begin{array}{ll}
w_{k}^{T} & v_{k}^{T}
\end{array}\right)\right]=\left(\begin{array}{cc}
Q & S \\
S^{T} & R
\end{array}\right)
$$

Only matrix $R$ depends on the variance of the measurement noise. Note that the measurement noise is denoted as $e$ in system (1), and the output noise term $v_{k}$ is in fact a sum of the measurement noise and the excitation noise in the case of acceleration measurements. In this case matrix $S$ only depends on the excitation noise, assuming that excitation and measurement noise are independent.

With these definitions, it can be seen that the expected value of the Hankel matrix does not depend on the measurement noise, since $R_{i}=\mathbf{E}\left(y_{k} y_{k-i}^{T}\right)=H F^{i-1} G$ for $i \geq 1$, where $G=F D H^{T}+S$ with $D$ being the state covariance. None of these quantities depend on the measurement noise under the previous assumptions.

However, the residual covariance $\Sigma^{e}=\lim _{N} \mathbf{E}\left(\left(\zeta_{N}^{e}-\mathbf{E} \zeta_{N}^{e}\right)\left(\zeta_{N}^{e}-\mathbf{E} \zeta_{N}^{e}\right)^{T}\right)$ depends on the measurement noise, since squared terms like $\operatorname{vec}\left(\hat{R}_{i}\right) \operatorname{vec}\left(\hat{R}_{i}\right)^{T}$ appear within the expectation, and the expected value of data correlations without lag, $\mathbf{E}\left(y_{k} y_{k}^{T}\right)=H D H^{T}+R$, indeed depends on the measurement noise [19]. However, we will not make a detailed mathematical analysis of the relationship between residual covariance and measurement noise in this paper, but content ourselves with a qualitative analysis for simplicity and clarity.

For the analysis of the effect of changes in the measurement noise between noise properties $e_{1}$ and $e_{2}$, we denote $e_{1}>e_{2}$ if the respective output noise covariance matrices satisfy $R^{e_{1}} \succ R^{e_{2}}$ (i.e. $R^{e_{1}}-R^{e_{2}}$ is positive definite). $e_{1}$ represents a higher measurement noise than $e_{2}$. This is the case if each of the measured signals in the first configuration have a lower signal to noise ratio than the respective signals in the second configuration (while the properties of the ambient excitation noise remain the same). A higher measurement noise leads to larger variations in the residual and thus to a bigger residual covariance. For our qualitative analysis, assume respectively $\Sigma^{e_{1}}=\alpha \Sigma^{e_{2}}$ with a scalar magnification factor $\alpha>1$ to be able to study this 
effect in a closed form formulation. This magnification factor is in direct relation with the signal to noise ratio if the noise type is white. However, for colored noise this magnification factor is an approximate representation of the noise effect.

The effect of changes in the measurement noise is now investigated in two settings. In the first one, the noise properties between the reference state and possibly damaged state are constant, while in the second setting they are different.

\subsubsection{Equal noise properties between the reference state and possibly damaged state}

In this section, it is assumed that the measurement noise properties in data from reference state and possibly damaged state are equal. We compare different noise properties that are the equal in both states. Note that the residual covariance matrices $\Sigma^{e_{1}}$ and $\Sigma^{e_{2}}$ for different noise properties $e_{1}$ and $e_{2}$ are assumed to be obtained from reference datasets under the respective conditions.

Theorem 3 If the structure is undamaged and the noise properties of both the reference state data and the current state data are equal, then an increase or decrease of the noise in both states does not change the expected $\chi^{2}$ value. In other words, $\mathbf{E}\left[\left(\zeta_{N}^{e_{1}}\right)^{T}\left(\Sigma^{e_{1}}\right)^{-1} \zeta_{N}^{e_{1}}\right]=\mathbf{E}\left[\left(\zeta_{N}^{e_{2}}\right)^{T}\left(\Sigma^{e_{2}}\right)^{-1} \zeta_{N}^{e_{2}}\right]$ under $H_{0}$

Proof 3 From the property of the $\chi^{2}$ distribution in (20) it follows that the expected value of the respective $\chi^{2}$ values is $d=\operatorname{dim}\left(\zeta_{N}^{e_{1}}\right)=\operatorname{dim}\left(\zeta_{N}^{e_{2}}\right)$ under $H_{0}$, as in proof 1 , which is independent of the noise.

Theorem 4 If the structure is damaged and the noise properties of both the reference state data and the current state data are equal, then an increase or decrease of the noise in both states results in a change (in inverse direction) in the expected $\chi^{2}$ value for a constant damage. In other words, if $e_{1}>e_{2}$ then $\mathbf{E}\left[\left(\zeta_{N}^{e_{1}}\right)^{T}\left(\Sigma^{e_{1}}\right)^{-1} \zeta_{N}^{e_{1}}\right]<\mathbf{E}\left[\left(\zeta_{N}^{e_{2}}\right)^{T}\left(\Sigma^{e_{2}}\right)^{-1} \zeta_{N}^{e_{2}}\right]$ under $H_{1}$.

Proof 4 As shown in the beginning of Section 4.2, the measurement noise does not influence the expected value of the respective Hankel matrices. Hence, $\delta=\mathbf{E} \zeta_{N}^{e_{1}}=\mathbf{E} \zeta_{N}^{e_{2}}$ is equal for both noise configurations (see also Eq. (21)), while the non-centrality parameters are $n_{c}^{e_{1}}=\delta^{T}\left(\Sigma^{e_{1}}\right)^{-1} \delta$ and $n_{c}^{e_{2}}=\delta^{T}\left(\Sigma^{e_{2}}\right)^{-1} \delta$, respectively. Due to assumption $\Sigma^{e_{1}}=\alpha \Sigma^{e_{2}}$ it follows $n_{c}^{e_{1}}=\frac{1}{\alpha} n_{c}^{e_{2}}$ with $\alpha>1$, hence $n_{c}^{e_{1}}<n_{c}^{e_{2}}$. Then, the assertion follows from property (20) of the $\chi^{2}$ distribution.

Theorem 4 is also intuitive in the sense that higher noise, i.e. a lower signal-to-noise ratio, decreases the quality of the data and makes it harder to detect damage, which is reflected in the lower $\chi^{2}$ test value.

\subsubsection{Different noise properties between the reference state and possibly damaged state}

In this section it is assumed that the measurement noise will change in the test data irrespective to the noise in the reference data where the residual covariance was computed. Note that since the noise properties of the residual do not correspond to its covariance anymore, one would need to recompute the covariance matrix to accommodate noise changes in a correct test [11]. Moreover, the resulting $\chi^{2}$ test value does not satisfy the stated parameters of the $\chi^{2}$ distribution as in Section 3 anymore, which are shifted now. However, the numerical computation of the covariance is complex and impractical on each tested dataset in practice. Hence, the covariance is usually only computed once in the reference state, which is valid when the measurement noise properties are stable. In this section we investigate the consequence of different noise properties on the test results, violating the theoretical assumptions of the test.

First, the effect of changes in the measurement noise in the test data are investigated, while the noise level in the residual covariance remains constant. Second, different noise levels in the residual covariance are investigated, while the noise level in the test data remains constant. 
Theorem 5 Change in the noise properties of the test data results in a change in the expected $\chi^{2}$ value in the same direction, regardless to the state of the structure. In other words, if $e_{1}>e_{2}$ then $\mathbf{E}\left[\left(\zeta_{N}^{e_{1}}\right)^{T}\left(\Sigma^{e_{1}}\right)^{-1} \zeta_{N}^{e_{1}}\right]>\mathbf{E}\left[\left(\zeta_{N}^{e_{2}}\right)^{T}\left(\Sigma^{e_{1}}\right)^{-1} \zeta_{N}^{e_{2}}\right]$ both under $H_{0}$ and $H_{1}$.

Proof 5 Analogous to Proof 4, if follows from the property (20) of the $\chi^{2}$ distribution $\mathbf{E}\left[\left(\zeta_{N}^{e_{1}}\right)^{T}\left(\Sigma^{e_{1}}\right)^{-1} \zeta_{N}^{e_{1}}\right]=d+\delta^{T}\left(\Sigma^{e_{1}}\right)^{-1} \delta$. Using $\quad \Sigma^{e_{1}}=\alpha \Sigma^{e_{2}}$ with $\quad \alpha>1, \quad$ it follows furthermore $\mathbf{E}\left[\left(\zeta_{N}^{e_{2}}\right)^{T}\left(\Sigma^{e_{1}}\right)^{-1} \zeta_{N}^{e_{2}}\right]=\frac{1}{\alpha} \mathbf{E}\left[\left(\zeta_{N}^{e_{2}}\right)^{T}\left(\Sigma^{e_{2}}\right)^{-1} \zeta_{N}^{e_{2}}\right]$. The right expectation corresponds now to a standard $\chi^{2}$ distribution and hence

$$
\begin{aligned}
\mathbf{E}\left[\left(\zeta_{N}^{e_{2}}\right)^{T}\left(\Sigma^{e_{1}}\right)^{-1} \zeta_{N}^{e_{2}}\right] & =\frac{1}{\alpha}\left(d+\delta^{T}\left(\Sigma^{e_{2}}\right)^{-1} \delta\right)=\frac{1}{\alpha}\left(d+\delta^{T}\left[\alpha\left(\Sigma^{e_{1}}\right)^{-1}\right] \delta\right) \\
& =\frac{1}{\alpha} d+\delta^{T}\left(\Sigma^{e_{1}}\right)^{-1} \delta
\end{aligned}
$$

Comparing now with $\mathbf{E}\left[\left(\zeta_{N}^{e_{1}}\right)^{T}\left(\Sigma^{e_{1}}\right)^{-1} \zeta_{N}^{e_{1}}\right]$, the assertion follows both for $H_{0}($ where $\delta=0)$ and for $H_{l}$, since $\alpha>1$.

Theorem 5 may be somewhat counterintuitive as it states "less noise leads to a weaker reaction of the test". However, this would not be the case if the appropriate covariance matrix had been used, which would be of lower magnitude and thus would normalize the residual correctly by dividing it with lower values.

Theorem 6 Regardless of the state of the system, change in the noise properties of the reference data, on which the residual covariance is computed, results in a change in inverse direction in the expected $\chi^{2}$ value. In other words, if $e_{1}>e_{2}$ then $\mathbf{E}\left[\left(\zeta_{N}^{e_{1}}\right)^{T}\left(\Sigma^{e_{1}}\right)^{-1} \zeta_{N}^{e_{1}}\right]<\mathbf{E}\left[\left(\zeta_{N}^{e_{1}}\right)^{T}\left(\Sigma^{e_{2}}\right)^{-1} \zeta_{N}^{e_{1}}\right]$ both under $H_{0}$ and $H_{l}$

Proof 6 The proof is analogous to the Proof 5. We have $\mathbf{E}\left[\left(\zeta_{N}^{e_{1}}\right)^{T}\left(\Sigma^{e_{1}}\right)^{-1} \zeta_{N}^{e_{1}}\right]=d+\delta^{T}\left(\Sigma^{e_{1}}\right)^{-1} \delta$, and since $\Sigma^{e_{1}}=\alpha \Sigma^{e_{2}}$ with $\alpha>1, \mathbf{E}\left[\left(\zeta_{N}^{e_{1}}\right)^{T}\left(\Sigma^{e_{2}}\right)^{-1} \zeta_{N}^{e_{1}}\right]=\alpha \mathbf{E}\left[\left(\zeta_{N}^{e_{1}}\right)^{T}\left(\Sigma^{e_{1}}\right)^{-1} \zeta_{N}^{e_{1}}\right]=\alpha d+\alpha \delta^{T}\left(\Sigma^{e_{1}}\right)^{-1} \delta$. Hence the assertion follows both for $H_{0}$ and for $H_{1}$.

\section{Numerical application}

In this section the theorems stated at section 4 are demonstrated for a simple mass-spring system. This system is composed of six degrees of freedom associated to six masses connected with springs as shown in Figure 1 . There is a damping ratio of $2 \%$ associated to all modes. Damage is modeled as a stiffness reduction of $5 \%$ of the second spring, i.e. $\mathrm{k}_{2}$. The excitation is simulated as Gaussian white noise and the resulting acceleration measurements are acquired from three sensors located on the masses at a sampling rate of $50 \mathrm{~Hz}$. In order to illustrate the effects in the theorems, three case studies are performed. The first one is related to theorems 1 to 2 , second one is related to theorems 3 to 4 , and the last one is related to theorems 5 to 6 .

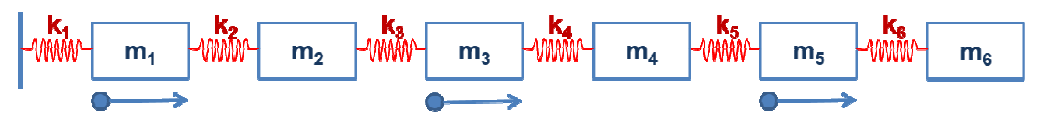

Fig 1 The mass-spring model and the sensor locations 


\subsection{Cases study 1 , effect of number of samples}

For this case, the number of samples is changed from 1000, 2000, .., to 10000 in 10 steps for both undamaged and damaged cases. In each step, 100 repetitions are made to calculate the mean, representing the expected value of the $\chi_{N}^{2}$. The measurement noise ratio in all cases is 5\%. It can be seen from the results in Figure 2, that as stated in theorem 1, when the model is not damaged, the expected $\chi_{N}^{2}$ is not changed. However, when the model is damaged, it can be seen that this value grows linearly with the number of samples, which confirms the (linear) factor $N$ in the non-centrality parameter as shown in the proof of Theorem 2 .

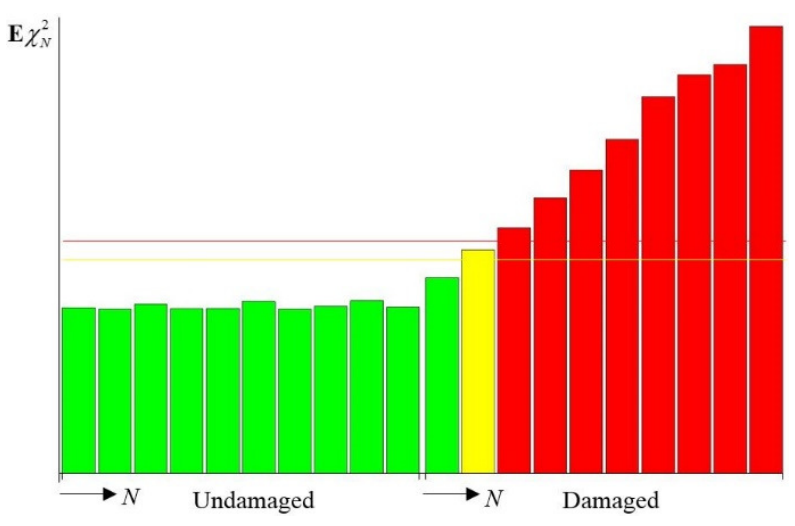

Fig 2 Expected $\chi_{N}^{2}$ value evaluated for different number of samples in damaged and undamaged conditions (red line: 99 percentile, yellow line: 95 percentile)

\subsection{Case study 2, effect of noise with Equal properties}

In here, the number of samples is kept constant at 10000. However, the measurement noise, which has equal properties in reference and testing state, is changed. This noise ratio is changed from 5\% to $125 \%$ in 25 steps for damaged and undamaged conditions. Again in each step the repetition is 100 times. The results are presented in Figure 3. The test values in the undamaged state are constant and independent of the noise ratio, confirming Theorem 3 . The test values in the damaged state decrease when the noise ratio increases, as shown in Theorem 4. From Figure 3 it can be observed that the test values decrease approximately quadratically with increasing noise, thus the factor $\alpha$ in Section 3.2 seems to be quadratic in the measurement noise level.

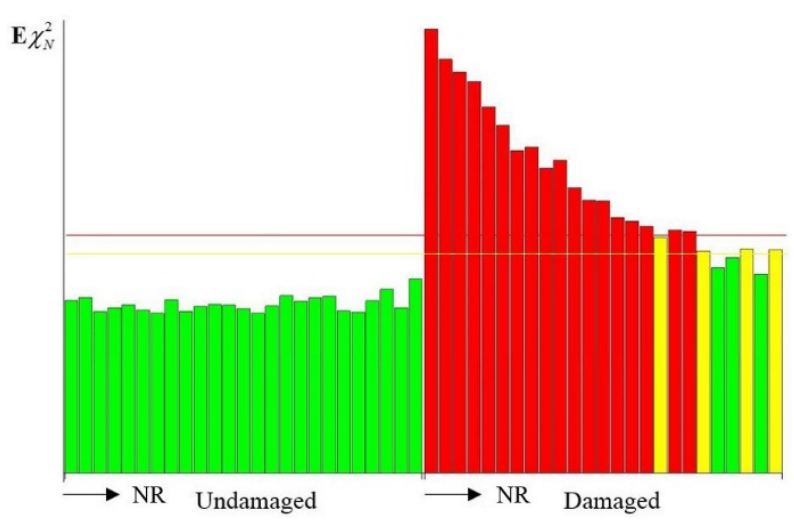

Fig 3 Expected $\chi_{N}^{2}$ value evaluated for different noise ratios with equal properties in damaged and undamaged conditions (red line: 99 percentile, yellow line: 95 percentile)

\subsection{Case study 3, effect of noise with unequal properties}

In this case study, the noise properties are not equal in the reference and test states as mentioned in section 4.2. For this purpose, same as previous case study, the number of samples is constantly equal to 10000. The noise is being increased in 25 steps from 
$5 \%$ to $125 \%$ with 100 repetitions in each step. This is also investigated for damaged and undamaged conditions. In Figure 4 the results are shown when the measurement noise is changed only for the testing state (both in undamaged and damaged conditions, respectively). The measurement noise in the reference state that was used to set up the residual covariance is constant at 5\%. It can be seen that both in undamaged and damaged states the test value increases when the noise level increases, confirming Theorem 5. Again, the increase rate seems to be quadratic.

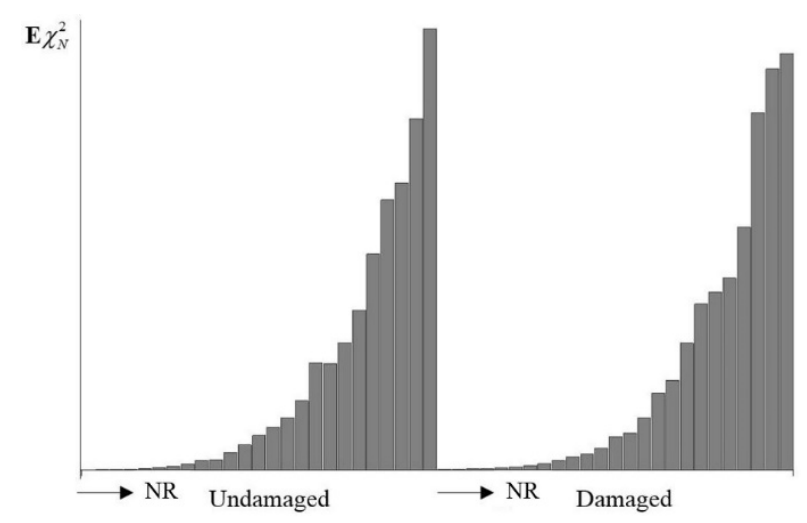

Fig 4 Expected $\chi_{N}^{2}$ value evaluated for different noise ratios only in the test data, in damaged and undamaged conditions

In Figure 5, the same study is done for the changing of measurement noise in the residual covariance computed in the reference state while the measurement noise in the test data is kept constant at 5\%. It can be seen that by increasing the measurement noise in the reference data, the expected $\chi_{N}^{2}$ value is being decreased for both undamaged and damaged conditions, as stated in Theorem 6.

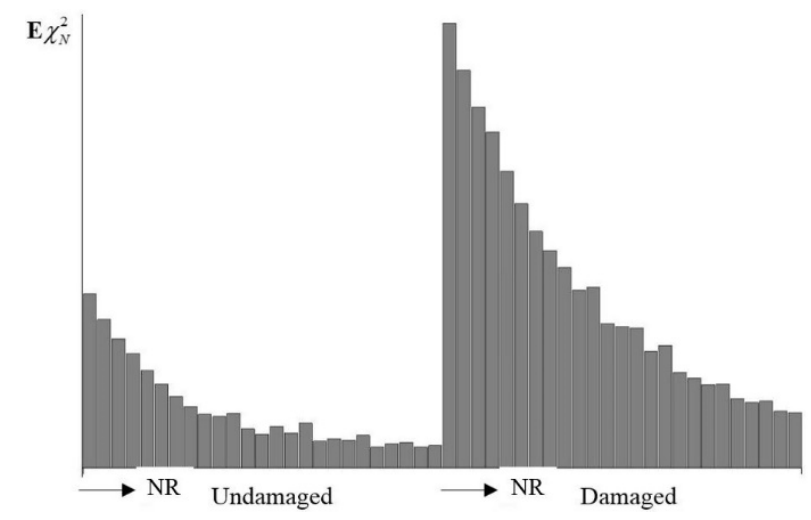

Fig 5 Expected $\chi_{N}^{2}$ value evaluated for different noise ratios only in the reference data, in damaged and undamaged conditions

\section{Discussion and conclusion}

In this paper, several theorems are proposed and proved on the effect of noise and number of samples on SSDD technique. From these theorems some conclusions can be inferred that will serve the user of SSDD technique as a guideline in dealing with these effects.

I) Considering theorems 1 and 2, data duration does not affect the expected $\chi^{2}$ value in the reference state. This is an advantage of this approach that will help in identifying a unique threshold in the reference state which can be compared to the $\chi^{2}$ value acquired from the test data. However, when the structure is damaged, by increasing the data length the $\chi^{2}$ value will be increased. In other words, if there is more data the damage state becomes more distinct and identifiable. Therefore, the more samples we have, the better results we get to detect the damage, and if there is not enough data, the damage state may not be identifiable.

II) Different noise levels in the system (each time both for reference and test data) lead to changes in the resulting $\chi^{2}$ values acquired from damaged structure changes in inverse direction (theorems 3 and 4). Therefore, an increase of measurement noise in the system results in decreasing the $\chi^{2}$ value for damaged state, making the damage possibly undetectable. Note 
that this can be compensated by longer datasets (see previous point). The amount of measurement noise in the system should not be too high.

III) It can be inferred from theorem 5 that by having higher noise in the test or validation data, while the residual covariance is not re-evaluated, their $\chi^{2}$ value becomes higher. This can affect the damage detection process in two ways. Firstly, if the safety thresholds are evaluated from a low noise reference data, then a high noise test data from undamaged structure can be identified as being damaged leading to a false alarm. Secondly, if the safety thresholds are evaluated from a high noise reference data and the noise in test data of damaged structure is very low, then the damaged structure might not be detected. These two suggests that the measurement noise in the reference and test data should be about the same. The sensitivity of the threshold was studied in [15].

IV) Based on theorem 6, increasing noise in the reference data results in a decrease of the $\chi^{2}$ value for both undamaged and damaged test data. Nevertheless, the effect of damage for the same noise level is still visible.

It should be mentioned that, in all of these cases it is assumed that the reference data is not corrupted with too high noise and that the left null-space $S_{0}^{T}$ and the residual covariance matrix $\Sigma^{e}$ are evaluated properly.

\section{References}

[1] Fan W, and Pizhong Q. "Vibration-based damage identification methods: a review and comparative study." Structural Health Monitoring 10.1, 83-111, 2011.

[2] Doebling SW, Farrar CR, Prime MB. "A summary review of vibration-based damage identification methods." Shock and vibration digest 30.2, 91-105, 1998.

[3] Salawu OS. "Detection of structural damage through changes in frequency: a review." Engineering structures, 19.9, 718$723,1997$.

[4] Worden K, Farrar CR, Manson G, Park G. "The fundamental axioms of structural health monitoring." Proceedings of the Royal Society of London A: Mathematical, Physical and Engineering Sciences. 463.2082. The Royal Society, 2007.

[5] Farrar CR, Doebling SW, Nix DA. "Vibration-based structural damage identification." Philosophical Transactions of the Royal Society of London A: Mathematical, Physical and Engineering Sciences359.1778, 131-149, 2001.

[6] Yan AM, De Boe P, Golinval JC. "Structural damage diagnosis by Kalman model based on stochastic subspace identification."Structural Health Monitoring 3.2, 103-119, 2004.

[7] Worden K, Manson G, Fieller NRJ. "Damage detection using outlier analysis." Journal of Sound and Vibration 229.3, 647-667, 2000 .

[8] Basseville M, Abdelghani M, Benveniste A. "Subspace-based fault detection algorithms for vibration monitoring", Automatica 36.1, 2000, pp. 101-109.

[9] Basseville M, Mevel L, Goursat M. "Statistical model-based damage detection and localization: subspace-based residuals and damage-to-noise sensitivity ratios", Journal of Sound and Vibration, 275. 3, 769-794, 2004.

[10] Carden EP, Fanning P. "Vibration based condition monitoring: a review." Structural health monitoring 3.4, 355-377, 2004.

[11]Döhler M, Mevel L, Hille F. "Subspace-based damage detection under changes in the ambient excitation statistics." Mechanical Systems and Signal Processing 45.1, 207-224, 2014.

[12]Döhler M, Hille F. "Subspace-based damage detection on steel frame structure under changing excitation." Structural Health Monitoring, Volume 5. Springer International Publishing, 2014. 167-174.

[13] Alvandi A, Cremona C. "Assessment of vibration-based damage identification techniques." Journal of sound and vibration 292.1, 179-202, 2006.

[14] Döhler M, Mevel L. "Subspace-based fault detection robust to changes in the noise covariances." Automatica 49.9 (2013): 2734-2743.

[15] Allahdadian S, Ventura C, Andersen P, Mevel L, Döhler M. "Investigation on the sensitivity of subspace based damage detection technique to damage and noise levels." IOMAC-International Operational Modal Analysis Conference, 2015. 
[16] Allahdadian S, Ventura C, Andersen P, Mevel L, Döhler M. "Subspace based damage detection technique: investigation on the effect of number of samples", CCEE-Canadian Conference on Earthquake Engineering, 2015.

[17] Allahdadian S, Ventura C, Andersen P, Mevel L, Döhler M. "Sensitivity Evaluation of Subspace-based Damage Detection Method to Different Types of Damage." Structural Health Monitoring and Damage Detection, Volume 7. Springer International Publishing, 11-18, 2015.

[18] Basseville M, Benveniste A, Goursat M, Hermans L, Mevel L. Van der Auweraer H. "Output-only subspace-based structural identification: from theory to industrial testing practice." Journal of Dynamic Systems, Measurement, and Control 123.4, 668-676, 2001.

[19] Van Overschee P, De Moor B. Subspace Identification for Linear Systems: Theory, Implementation, Applications. Kluwer, 1996.

[20]Benveniste A, Basseville M, Moustakides G. "The asymptotic local approach to change detection and model validation." IEEE Transactions on Automatic Control 32.7, 583-592, 1987.

[21] Balmès E, Basseville M, Bourquin F, Mevel L, Nasser H, Treyssède F. "Merging sensor data from multiple temperature scenarios for vibration-based monitoring of civil structures." Structural Health Monitoring, 7(2):129-142, 2008. 